\title{
A SINGULAR PERTURBATION NONLINEAR BOUNDARY VALUE PROBLEM AND THE E-CONDITION FOR A SCALAR CONSERVATION LAW
}

\author{
$\mathrm{BY}$ \\ JIANG JIE (Jilin University, Changchun, JL 130023, P.R. China) \\ AND \\ WANG XUEKONG (Daqing Petroleum Institute, P.R. China)
}

Abstract. This paper deals with the singular perturbation boundary value problem

$$
\left\{\begin{array}{l}
\varepsilon\left(k(v(s)) v^{\prime}(s)\right)^{\prime}+(s g(v(s))-\varphi(v(s))) v^{\prime}(s)+f(v(s))=0 \quad \text { in } R, \\
v(-\infty)=A, \quad v(+\infty)=B ; \quad \varepsilon \geq 0, \quad A<B
\end{array}\right.
$$

whose solution $v_{\varepsilon}(s)$ is constructed by the aid of the solution $w_{\varepsilon}(t)$ to the two-point boundary value problem

$$
\left\{\begin{array}{l}
-\left(\frac{w^{\prime}(t)-\varphi(t)+\varepsilon f(t) k(t) / w(t)}{g(t)}\right)^{\prime}=\frac{\varepsilon k(t)}{w(t)} \text { in }(A, B), \\
w(A)=0, \quad w(B)=0 .
\end{array}\right.
$$

The restrictions on $\varphi(t), g(t), k(t)$, and $f(t)$ not only ensure that the two-point boundary value problem has a solution $w_{\varepsilon}(t)$ but also guarantee that as $\varepsilon$ tends to zero the solution $w_{\varepsilon}(s)$ pointwise converges to

$$
v_{0}(s)=A+(B-A) H\left(s-\frac{\Phi(B)}{G(B)}\right), \quad s \in R,
$$

the solution to the reduced problem, where $H(s)$ is the multiple-valued Heaviside function, $G(t)=: \int_{A}^{t} g(s) d s$, and $\Phi(t)=: \int_{A}^{t} \varphi(s) d s$. Moreover, the function $u_{\varepsilon}(x, t)=: v_{\varepsilon}(x / t)$, as a solution to the Riemann problem

$$
\left\{\begin{array}{l}
\frac{\partial G(u)}{\partial t}+\frac{\partial \Phi(u)}{\partial x}=\frac{f(u)}{t}+\varepsilon t \frac{\partial}{\partial x}\left(k(u) \frac{\partial u}{\partial x}\right), \quad x \in R, \quad t>0, \\
u(x, 0)=A+(B-A) H(x), \quad \text { for } x \in R
\end{array}\right.
$$

pointwise converges to $u_{0}(x, t)=: v_{0}(x / t)$, the discontinuous solution of the Riemann problem for the scalar conservation law $(\varepsilon=0)$. Obviously, $u_{0}(x, t)$ satisfies the classical Rankine-Hugoniot condition on the line of discontinuity $x=$ $t \Phi(B) / G(B)$, and the restriction on $\Phi(u)$ and $G(u)$,

$$
\Phi(u)-G(u) \Phi(B) / G(B) \geq 0 \text { on }[A, B],
$$

Received February 5, 1991.

1991 Mathematics Subject Classification. Primary 34B, 34D15, 35L65.

(C) 1992 Brown University 
is exactly the E-Condition proposed first by Oleinik. The technical arguments, which involve only the use of the Schauder Fixed Point Theorem and integral representations, are elementary.

1. Introduction. In this paper we deal with a nonlinear boundary value problem of the form

$$
\left\{\begin{array}{r}
\varepsilon\left(k(v(s))^{\prime} v(s)\right)^{\prime}+(s g(v(s))-\varphi(s)) v^{\prime}(s)+f(v(s))=0 \quad \text { in } R \\
v(-\infty)=A, \quad v(+\infty)=B
\end{array}\right.
$$

where $\varepsilon \geq 0$ is a small parameter, $A, B, A<B$, are given real numbers, and the functions $f(t), k(t), g(t)$, and $\varphi(t)$ satisfy the following conditions:

(I) Both $f(t)$ and $k(t)$ are real-valued continuous functions defined on [A,B], $f(A)=f(B)=0, k(t)>0$ a.e. on $[A, B]$, and $G(t)=: \int_{A}^{t} g(s) d s$ and $\Phi(t)=$ : $\int_{A}^{t} \varphi(s) d s$ are both absolutely continuous functions defined on $[A, B], G(t)$ is strictly increasing on $[A, B]$.

(II) $w_{0}(t)=: \Phi(t)-G(t) \Phi(B) / G(B) \geq 0$ on $[A, B],-(G(B)-G(t)) \leq f(t) \leq 0$ or $0 \leq f(t) \leq G(t)$ on $[A, B]$, and there is a positive number $M$ such that $k(t)|f(t)| \leq$ $M g(t)$ a.e. on $[A, B]$.

$(\text { II })^{\prime} \quad w_{0}(t)=: \Phi(t)-G(t) \Phi(B) / G(B)>0$ a.e. on $[A, B],-(G(B)-G(t)) \leq f(t) \leq$ $G(t)$ on $[A, B]$, and $k(t) / w_{0}(t)$ is integrable on $[A, B]$.

A solution $v_{\varepsilon}(s)$ to the boundary value problem $(1)_{\varepsilon}-(2)$ can be constructed by the aid of the solution $w_{\varepsilon}(t)$ to the two-point boundary value problem

$$
\left\{\begin{array}{l}
-\left(\frac{w^{\prime}(t)-\varphi(t)+\varepsilon f(t) k(t) / w(t)}{g(t)}\right)^{\prime}=\frac{\varepsilon k(t)}{w(t)} \text { in }(A, B), \\
w(A)=0, \quad w(B)=0 .
\end{array}\right.
$$

The hypotheses (I) and (II) or (I) and (II) not only ensure that the two-point boundary value problem $(3)_{\varepsilon}-(4)_{0}$ has a solution $w_{\varepsilon}(t)$ but also guarantee that the solution $v_{\varepsilon}(s)$ pointwise converges to

$$
v_{0}(s)=A+(B-A) H\left(s-\frac{\Phi(B)}{G(B)}\right), \quad s \in R,
$$

a solution to the reduced problem $(1)_{0}-(2)$, where $H(s)=0$ for $s<0, H(s)=1$ for $s>0$, and $H(0)=[0,1]$.

The foregoing problem arises in the search of similarity solutions of the Riemann problem

$$
\begin{aligned}
& \frac{\partial G(u)}{\partial t}+\frac{\partial \Phi(u)}{\partial x}=\frac{f(u)}{t}+\varepsilon t \frac{\partial}{\partial x}\left(k(u) \frac{\partial u}{\partial x}\right), \quad x \in R, t>0, \\
& u(x, 0)=A+(B-A) H(x) .
\end{aligned}
$$

A special case of the Riemann problem $(5)_{0}-(6)$, where $G(u) \equiv u$ and $f(u) \equiv 0$, has been studied by many authors. For details, see, for example, Proposition 2.1 in [1], Theorem 2.2 in [2], [5], pp. 301-303, and [6].

It is easy to see that the function $u_{\varepsilon}(x, t)=: v_{\varepsilon}(x / t)$ is a solution to the Riemann problem $(5)_{\varepsilon}-(6)$ and pointwise converges to $u_{0}(x, t)=: v_{0}(x / t)$, the discontinuous 
solution to the Riemann problem $(5)_{0}-(6)$. Obviously, $u_{0}(x, t)$ satisfies the classical Rankine-Hugoniot condition on the line of discontinuity, $x=t \Phi(B) / G(B)$, while

$$
\Phi(u)-G(u) \Phi(B) / G(B) \geq 0 \text { on }[A, B]
$$

is exactly the E-condition proposed first by Oleinik [3].

The plan of this paper is as follows. In Sec. 2 we convert the nonlinear boundary value problem $(1)_{\varepsilon}-(2)$ into the two-point boundary value problem $(3)_{\varepsilon}-(4)_{0}$. Sec. 3 is devoted to the two-point boundary value problem $(3)_{\varepsilon}-(4)_{0}$. In Sec. 4 we construct a solution $v_{\varepsilon}(s)$ utilizing the solution $w_{\varepsilon}(t)$ to the two-point boundary value problem $(3)_{\varepsilon}-(4)_{0}$. The last section is concerned with the Riemann problem $(5)_{\varepsilon}-(6)$.

2. Formal reduction. In this section, we (formally) convert the boundary value problem $(1)_{\varepsilon}-(2)$ into the two-point boundary value problem $(3)_{\varepsilon}-(4)_{0}$.

By a solution to the boundary value problem $(1)_{\varepsilon}-(2)$ with $\varepsilon>0$, we shall mean the function $v_{\varepsilon}(s)$ satisfying the following conditions:

(i) $v_{\varepsilon}(s)$ is an increasing, absolutely continuous function defined on $R=(-\infty, \infty)$ with $v_{\varepsilon}(-\infty)=A$ and $v_{\varepsilon}(+\infty)=B$,

(ii) $k\left(v_{\varepsilon}(s)\right) v_{\varepsilon}^{\prime}(s)$ is equivalent to an absolutely continuous function defined on $R$, and

(iii) the equality $(1)_{\varepsilon}$ holds almost everywhere on $R$.

If the solution $v_{\varepsilon}(s)$ converges to a limit, denoted by $v_{0}(s)$, pointwise on $R$, as $\varepsilon$ tends to zero, the $v_{0}(s)$ is said to be a solution to the reduced boundary value problem $(1)_{0}-(2)$.

Let $t=v(s)$ be a solution to the boundary value problem $(1)_{\varepsilon}-(2)$. If $v(s)$ is still strictly increasing on $R$, then $v(-\infty)=v(+\infty)=0$ and the function $s=y(t)$, inverse to $t=v(s)$, exists, and furthermore, $t=v(y(t))$ holds in $(A, B)$ and $v^{\prime}(y(t))=1 / y^{\prime}(t)>0$ a.e. in $(A, B)$.

Substituting $s=y(t)$ into the equation $(1)_{\varepsilon}$ and then putting $w(t)=\varepsilon k(t) / y^{\prime}(t)$, we obtain the two-point boundary value problem $(3)_{\varepsilon}-(4)_{0}$.

3. Two-point boundary value problem. In this section we study the two-point boundary value problem $(3)_{\varepsilon}-(4)_{0}$.

As the two endpoints $t=A$ and $t=B$ are singular for the problem, we need to consider the two-point boundary value problem, namely,

$$
\left\{\begin{array}{l}
-\left(\frac{w^{\prime}(t)-\varphi(t)+\varepsilon f(t) k(t) / w(t)}{g(t)}\right)^{\prime}=\varepsilon \frac{k(t)}{w(t)} \quad \text { in }(A, B), \\
w(A)=h, \quad w(B)=h ; \quad h>0 .
\end{array}\right.
$$

A function $w(t)$ will be called a solution to the equation $(3)_{\varepsilon}(\varepsilon>0)$ on $[A, B]$, if it is defined, absolutely continuous on $[A, B]$ and positive a.e. in $(A, B)$, and for any subinterval $[a, b]$ of $[A, B]$ it can be represented by the formula

$$
\begin{aligned}
w(t)= & \varepsilon \int_{a}^{b} J_{a b}(t, s) \frac{k(s)}{w(s)} d s+\frac{\varepsilon(G(t)-G(a))}{G(b)-G(a)} \int_{t}^{b} \frac{f(s) k(s)}{w(s)} d s \\
& -\frac{\varepsilon(G(b)-G(t))}{G(b)-G(a)} \int_{a}^{t} \frac{f(s) k(s)}{w(s)} d s+E(t)+\Phi(t),
\end{aligned}
$$


where

and

$$
J_{a b}(t, s)=: \begin{cases}(G(b)-G(t))(G(s)-G(a)) /(G(b)-G(a)) & \text { for } a \leq s \leq t \\ (G(b)-G(s))(G(t)-G(a)) /(G(b)-G(a)) & \text { for } t \leq s \leq b\end{cases}
$$

$$
E(t)=: \frac{(w(a)-\Phi(a))(G(b)-G(t))+(w(b)-\Phi(b))(G(t)-G(a))}{G(b)-G(a)} .
$$

In particular, a solution to the two-point boundary value problem $(3)_{\varepsilon}-(4)_{h}$ with $\varepsilon \geq 0$ and $h \geq 0, w(t)$ can be represented by the formula

$$
\begin{aligned}
w(t)= & h+w_{0}(t)+\varepsilon \int_{A}^{B} J_{A B}(t, s) \frac{k(s)}{w(s)} d s+\frac{\varepsilon G(t)}{G(B)} \int_{t}^{B} \frac{f(s) k(s)}{w(s)} d s \\
& -\frac{\varepsilon(G(B)-G(t))}{G(B)} \int_{A}^{t} \frac{f(s) k(s)}{w(s)} d s \\
= & h+w_{0}(s)+\frac{\varepsilon G(t)}{G(B)} \int_{t}^{B} \frac{(G(B)-G(s)+f(s)) k(s)}{w(s)} d s \\
& +\frac{\varepsilon(G(B)-G(t))}{G(B)} \int_{A}^{t} \frac{(G(s)-f(s)) k(s)}{w(s)} d s \\
= & :(T w)(t),
\end{aligned}
$$

where

$$
w_{0}(t)=: \Phi(t)-G(t) \Phi(B) / G(B), \quad t \in[A, B],
$$

is clearly the unique solution to the two-point boundary value problem $(3)_{0}-(4)_{0}$. Moreover,

$$
\begin{aligned}
y_{\varepsilon}(t) & =:-\frac{w_{\varepsilon}^{\prime}(t)-\varphi(t)+\varepsilon f(t) k(t) / w_{\varepsilon}(t)}{g(t)} \\
& =\frac{\Phi(B)}{G(B)}+\frac{\varepsilon}{G(B)}\left(\int_{A}^{t} \frac{(G(s)-f(s)) k(s)}{w_{\varepsilon}(s)} d s-\int_{t}^{B} \frac{(G(B)-G(s)+f(s)) k(s)}{W_{\varepsilon}(s)} d s\right)
\end{aligned}
$$

is locally absolutely continuous and strictly increasing in $(A, B)$ when $\varepsilon>0$, and

$$
y_{0}(t)=:-\left(w_{0}(t)-\varphi(t)\right) / g(t)=\Phi(B) / G(B) \text { for } t \in(A, B)
$$

is a constant.

We are now in a position to prove the existence and uniqueness of the solution $w_{\varepsilon}(t)$ under the hypotheses (I) and (II).

LEMmA 1. Suppose that the hypotheses (I) and (II) hold; then for each $\varepsilon>0$ and each $h>0$, the problem $(3)_{\varepsilon}-(4)_{h}$ has at least one solution $w(t ; \varepsilon, h) \geq h$.

Proof. Define a mapping $T: W \rightarrow W$ by the right-hand side of $(8)_{h}^{\varepsilon}$, where $W=:\{w(s) \in C[A, B] ; h \leq w(s) \leq(T h)(s)\}$ and $C[A, B]$ is the set of all realvalued continuous functions defined on $[A, B]$.

By hypotheses (I) and (II), it is readily verified that $T$ is a compactly continuous mapping from $W$ into $W$. The Schauder Fixed Point Theorem tells us that the mapping $T$ has at least one fixed point, denoted by $w(t ; \varepsilon, h)$, in the set $W$. Obvicusly, $w(t ; \varepsilon, h) \geq h$ is a solution to the problem $(3)_{\varepsilon}-(4)_{h}$. 
LEMMA 2. If $h_{1}>h_{2}>0, \varepsilon \geq 0$, then

$$
0 \leq w\left(t ; \varepsilon, h_{1}\right)-w\left(t ; \varepsilon, h_{2}\right) \leq h_{1}-h_{2} \text { on }[A, B] .
$$

Proof. When $\varepsilon=0$, the lemma is clearly true, so it is enough to prove the lemma in the case of $\varepsilon>0$. We now denote $w\left(t ; \varepsilon, h_{1}\right)$ and $w\left(t ; \varepsilon, h_{2}\right)$ by $w_{1}(t)$ and $w_{2}(t)$, respectively, and prove only the left inequality, i.e., $w_{1}(t)-w_{2}(t) \geq 0$ on $[A, B]$, since the right inequality follows from the left one by the formula $(8)_{h}^{\varepsilon}$.

If the left inequality is not true, then there will be a point $t=D$ in $(A, B)$ such that $w_{1}(D)-w_{2}(D)<0$. Further, there exists an interval $(a, b), A<a<D<b<$ $B$, such that $w_{1}(a)-w_{2}(a)=w_{1}(b)-w_{2}(b)=0$ and $w_{1}(t)-w_{2}(t)<0$ in $(a, b)$ because $w_{1}(A)-w_{2}(A)=w_{1}(B)-w_{2}(B)=h_{1}-h_{2}>0$. Whence it follows by (7) that

$$
\begin{aligned}
0>w_{1}(t)-w_{2}(t)= & \varepsilon \int_{a}^{b} J_{a b}(t, s) k(s) R(s) d s+\frac{\varepsilon(G(t)-G(a))}{G(b)-G(a)} \int_{t}^{b} f(s) k(s) R(s) d s \\
& -\frac{\varepsilon(G(b)-G(t))}{G(b)-G(a)} \int_{a}^{t} f(s) k(s) R(s) d s
\end{aligned}
$$

i.e.,

$$
\begin{aligned}
& 0>\int_{t}^{b} f(s) k(s) R(s) d s-\frac{G(b)-G(t)}{G(b)-G(a)} \int_{a}^{b} f(s) k(s) R(s) d s, \\
& 0>\frac{G(t)-G(a)}{G(b)-G(a)} \int_{a}^{b} f(s) k(s) R(s) d s-\int_{a}^{t} f(s) k(s) R(s) d s
\end{aligned}
$$

for all $t \in(a, b)$, where $R(t)=1 / w_{1}(t)-1 / w_{2}(t)>0$ in $(a, b)$ and $R(a)=R(b)=$ 0 . When $0 \leq f(s) \leq G(s)$, (11) can be written as

$$
\begin{aligned}
0 & \leq \frac{\int_{a}^{b} f(s) k(s) R(s) d s}{G(b)-G(a)}<\frac{\int_{a}^{t} f(s) k(s) R(s) d s}{G(t)-G(a)} \\
& \leq \frac{\int_{a}^{t} M g(s) R(s) d s}{G(t)-G(a)} \leq M \max _{a \leq s \leq t} R(s) \text { for } t \in(a, b) .
\end{aligned}
$$

Letting $t \rightarrow a$ in the above yields $\int_{a}^{b} f(s) k(s) R(s) d s=0$ and hence $0>w_{1}(t)-$ $w_{2}(t) \geq 0$ in $(a, b)$ which is absurd. When $0 \geq f(s) \geq-(G(B)-G(s))$, the argument is similar and thus is omitted here.

In the same way, we can show the following two statements.

LEMMA 3. If $\varepsilon_{1}>\varepsilon_{2}>0$ and $h>0$, then

$$
0 \leq w\left(t ; \varepsilon_{1}, h\right)-w\left(t ; \varepsilon_{2}, h\right) \leq N\left(\varepsilon_{1}-\varepsilon_{2}\right) / h \text { on }[A, B],
$$

where $N=2 G(B) \int_{A}^{B} k(s) d s$.

Lemma 4. For each fixed $\varepsilon \geq 0$ and each fixed $h \geq 0$, the problem $(3)_{\varepsilon}-(4)_{h}$ has at most one solution.

Lemma 5. For each $\varepsilon>0$ the problem $(3)_{\varepsilon}-(4)_{0}$ has a solution $w(t ; \varepsilon, 0)$.

Proof. According to Lemma 2, the solution $w(t ; \varepsilon, h)$ converges to a limit, denoted by $w(t ; \varepsilon, 0)$, uniformly on $[A, B]$, as $h \rightarrow 0_{+}$. Inserting $w(t ; \varepsilon, h)$ into 
$(8)_{h}^{\varepsilon}$ and then letting $h \rightarrow 0_{+}$gives $(8)_{0}^{\varepsilon}$, by the Monotone Convergence Theorem. The equality $(8)_{0}^{\varepsilon}$ shows that the function $w(t ; \varepsilon, 0)$ is a solution to the problem $(3)_{\varepsilon}-(4)_{0}$.

Lemma 6 . As $\varepsilon \rightarrow 0$, the solution $w_{\varepsilon}(t)=: w(t ; \varepsilon, 0)$ converges to $w_{0}(t)$ uniformly on $[A, B]$ and $y_{\varepsilon}(t)$ converges to $y_{0}(t)$ uniformly on $[A+\delta, B-\delta]$ for any $2 \delta \in$ $(0, B-A)$.

Proof. Using Lemma 2, we have

$$
w(t ; 0,0) \leq w(t ; 0, h) \leq w(t ; 0,0)+h \text { on }[A, B] .
$$

By virtue of Lemma 3, we obtain

$$
w(t ; 0, h) \leq w(t ; \varepsilon, h) \leq w(t ; 0, h)+N \varepsilon / h \text { on }[A, B] .
$$

Hence, putting $\varepsilon=h^{2}$, we get, for $t$ on $[A, B]$,

$$
0 \leq w(t ; 0,0) \leq w\left(t ; h^{2}, 0\right) \leq w\left(t ; h^{2}, h\right) \leq w(t ; 0,0)+h(1+N) .
$$

This shows that $w(t ; \varepsilon, 0)$ converges to $w(t ; 0,0)$ uniformly on $[A, B]$ as $\varepsilon=h^{2}$ tends to zero.

For any $2 \delta \in(0, B-A)$ it follows from $(8)_{0}^{\varepsilon}$ and $(9)_{\varepsilon}$ that

$$
\left|y_{\varepsilon_{1}}(t)-y_{\varepsilon_{2}}(t)\right| \leq\left(w_{\varepsilon_{1}}(t)-w_{\varepsilon_{2}}(t)\right) / m_{\delta} \text { on }[A+\delta, B-\delta],
$$

where $\varepsilon_{1}>\varepsilon_{2}>0$ and $m_{\delta}=: \min \{G(A+\delta), G(B)-G(B-\delta)\}$. This shows that $y_{\varepsilon}(t)$ converges to $y_{0}(t)$ uniformly on $[A+\delta, B-\delta]$ as $\varepsilon$ tends to zero, because the first assertion is true. The proof is complete.

We can summarize the above results in the following statement.

THEOREM 1. Under hypotheses (I) and (II), the two-point boundary value problem $(3)_{\varepsilon}-(4)_{0}$ with $\varepsilon \geq 0$ has a unique solution $w_{\varepsilon}(t)$;

$$
y_{\varepsilon}(t)=:-\frac{w_{\varepsilon}^{\prime}(t)-\varphi(t)+\varepsilon f(t) k(t) / w_{\varepsilon}(t)}{g(t)}
$$

is locally absolutely continuous and strictly increasing in $(A, B)$ when $\varepsilon>0$ and

$$
y_{0}(t)=:-\frac{w_{0}^{\prime}(t)-\varphi(t)}{g(t)}=\frac{\Phi(B)}{G(B)} .
$$

Moreover, as $\varepsilon$ tends to zero, $w_{\varepsilon}(t)$ converges to $w_{0}(t)$ uniformly on $[A, B]$ and $y_{\varepsilon}(t)$ converges to $y_{0}(t)$ uniformly on $[A+\delta, B-\delta]$ for any $2 \delta \in(0, B-A)$.

Now we take up the problem $(3)_{\varepsilon}-(4)_{0}$ under the hypotheses (I) and (II) ${ }^{\prime}$. Our result is the following.

Theorem 2. Under the hypotheses (I) and (II $)^{\prime}$, for each fixed $\varepsilon>0$ the two-point boundary value problem $(3)_{\varepsilon}-(4)_{0}$ has at least one solution $w_{\varepsilon}(t)$. Moreover, as $\varepsilon$ tends to zero, $w_{\varepsilon}(t)$ converges to $w_{0}(t)$ uniformly on $[A, B]$ and $y_{\varepsilon}(t)$ converges to $y_{0}(t)$ uniformly on $[A+\delta, B-\delta]$ for any $2 \delta \in(0, B-A)$. 
Proof. Let

$$
\begin{aligned}
(M w)(t)=: & w_{0}(t)+\int_{A}^{t} \frac{(G(B)-G(t))(G(s)-f(s)) \varepsilon k(s)}{G(B) W(s)} d s \\
& +\int_{t}^{B} \frac{(G(B)-G(s)+f(s)) G(t) \varepsilon k(s)}{G(B) w(s)} d s
\end{aligned}
$$

where $w(s) \in X=:\left\{w(s) \in C[A, B] ; w_{0}(s) \leq w(s) \leq\left(M w_{0}\right)(s)\right\}$.

By hypotheses (I) and (II) ${ }^{\prime}$, it is easy to check that $M$ is a compactly continuous mapping from $X$ into $X$. An application of the Schauder Fixed Point Theorem shows that the mapping $M$ has at least one fixed point, denoted by $w_{\varepsilon}(t)$, in the set $X$. Clearly $w_{\varepsilon}(t) \geq w_{0}(t)$ is a solution to the problem $(3)_{\varepsilon}-(4)_{0}$.

By hypothesis $(\mathrm{II})^{\prime}$, it follows from $(8)_{0}^{\varepsilon}$ that

$$
\begin{aligned}
0 \leq w_{\varepsilon}(t)-w_{0}(t)= & \int_{A}^{t} \frac{(G(B)-G(t))(G(s)-f(s)) \varepsilon k(s)}{G(B) w_{\varepsilon}(s)} d s \\
& +\int_{t}^{B} \frac{(G(B)-G(s)+f(s)) G(t) \varepsilon k(s)}{G(B) w_{\varepsilon}(s)} d s \\
\leq & \varepsilon G(B) \int_{A}^{B} \frac{k(s)}{w_{0}(s)} d s, \quad \text { for } t \in[A, B]
\end{aligned}
$$

Therefore, as $\varepsilon$ tends to zero, $w_{\varepsilon}(t)$ converges to $w_{0}(t)$ uniformly on $[A, B]$ and $y_{\varepsilon}(t)$ to $y_{0}(t)$ uniformly on $[A+\delta, B-\delta]$ for any $2 \delta \in(0, B-A)$.

4. Constructing the solution to the problem $(1)_{\varepsilon}-(2)$. In this section we construct the solution $v_{\varepsilon}(s)$ to the boundary value problem $(1)_{\varepsilon}-(2)$, utilizing the solution $w_{\varepsilon}(t)$ of the two-point boundary value problem $(3)_{\varepsilon}-(4)_{0}$.

We need some propositions.

Proposition 1 (Corollary 4, [3]). Let $v(s)$ be an increasing function defined on $[a, b]$ and let $w(t)$ be an absolutely continuous function defined on $[v(a), v(b)]$. Then $w(v(s))$ has a finite derivative a.e. on $[a, b]$ and the chain rule

$$
\frac{d}{d s} w(v(s))=w^{\prime}(v(s)) v^{\prime}(s)
$$

holds a.e. on $[a, b]$.

Proposition 2 (Corollary 6, [3]). Let $v(s)$ be an increasing, absolutely continuous function defined on $[a, b]$ and let $w(t)$ be an integrable function defined on $[v(a), v(b)]$. Then $w(v(s)) v^{\prime}(s)$ is integrable on $[a, b]$ and the change of variables formula

$$
\int_{a}^{b} w(v(s)) v^{\prime}(s) d s=\bar{W}(v(b))-\bar{W}(v(a))
$$

holds, where $\bar{W}(t)$ is an indefinite integral of $w(t)$.

As a consequence of the above propositions, we have 
Proposition 3. Let $(a, b)$ be a finite open interval and $y(t)$ be a strictly increasing, locally absolutely continuous function defined on $(a, b)$. Then the function $t=$ $v(s)$, inverse to $s=y(t)$, is a strictly increasing, absolutely continuous function defined on $\left(s_{a}, s_{b}\right)$, where $s_{a}=: y(a+0)$ and $s_{b}=: y(b-0)$. Moreover $v\left(s_{a}-0\right)=a$ and $v\left(s_{b}+0\right)=b$.

Now we introduce the following definition.

DEFINITION. Let $s=y(t)$ be an increasing, locally absolutely continuous function defined on $(A, B)$. A function $t=v(s)$ is said to be a generalized inverse to the function $s=y(t)$, if it is defined on $R$, increasing, of bounded variation, and possibly multiple-valued, and its graph in the $(s, t)$-plane is a locally rectifiable continuous curve which is congruent with the graph of $s=y(t)$, provided that at each endpoint of the latter, if necessary, a half line parallel to the $s$-axis is joined to it.

By the definition,

$$
v\left(s_{A}\right)=: \lim _{s \rightarrow s_{A}} v(s)=A ; \quad v\left(s_{B}\right)=: \lim _{s \rightarrow s_{B}} v(s)=B,
$$

where $s_{A}=: y(A+0)$ and $s_{B}=: y(B-0)$. Clearly, $v(s)=A$ for $s s_{A}$ or $v(s)=B$ for $s \geq s_{B}$ when $s_{A}$ or $s_{B}$ is finite. For example, if

$$
y(t) \equiv \Phi(B) / G(B), \quad \text { in }(A, B),
$$

then

$$
v(s)=A+(B-A) H\left(s-\frac{\Phi(B)}{G(B)}\right),
$$

where $H(s)$ is the multiple-valued Heaviside function.

Let $w_{\varepsilon}(t)$ be the solution to the two-point boundary value problem $(3)_{\varepsilon}-(4)_{0}$, $\varepsilon \geq 0$. Then the function

$$
y_{\varepsilon}(t)=:-\frac{w_{\varepsilon}^{\prime}(t)-\varphi(t)+\varepsilon f(t) k(t) / w(t)}{g(t)}
$$

is increasing, locally absolutely continuous in $(A, B)$, by Theorem 1 or 2 . Consequently, the function $t=v_{\varepsilon}(s)$, generalized inverse to $s=y_{\varepsilon}(t)$, is defined on $R$, increasing, and of bounded variation, and $v_{\varepsilon}(-\infty)=A$ and $v_{\varepsilon}(+\infty)=B$. We now prove that the function $v_{\varepsilon}(s)$ is a solution to the boundary value problem $(1)_{\varepsilon}-(2)$ with $\varepsilon \geq 0$.

Lemma 7. $v_{\varepsilon}(s)$ converges to $v_{0}(s)$ pointwise on $R$ as $\varepsilon$ tends to zero.

Proof. The lemma is an immediate consequence of the definition of the function $v_{\varepsilon}(s), \varepsilon \geq 0$, and Theorem 1 or 2 .

LemMA 8. If $\varepsilon>0$, then $v_{\varepsilon}(s)$ is a solution to the boundary value problem $(1)_{\varepsilon}-(2)$.

Proof. Under the assumption of the lemma, we have

$$
y_{\varepsilon}^{\prime}(t)=\frac{\varepsilon k(t)}{w_{\varepsilon}(t)}
$$


is positive a.e. in $(A, B)$, i.e., $y_{\varepsilon}(t)$ is strictly increasing in $(A, B)$. Thus, the restriction of $v_{\varepsilon}(s)$ to $\left(s_{A}, s_{B}\right)$ is exactly the inverse function of $y_{\varepsilon}(t)$, where $s_{A}=$ : $y_{\varepsilon}(A+0)$ and $s_{B}=: y_{\varepsilon}(B-0)$. By virtue of Proposition 3, $v_{\varepsilon}(s)$ is strictly increasing and absolutely continuous in $\left(s_{A}, s_{B}\right)$; further, $v_{\varepsilon}(s)$ is absolutely continuous on $R$ independent of whether $s_{A}$ or $s_{B}$ is finite. It follows from $(9)_{\varepsilon}^{\prime}$ that

$$
w_{\varepsilon}(t)=\frac{\varepsilon k(t)}{y_{\varepsilon}^{\prime}(t)}
$$

holds a.e. in $(A, B)$. Since $w_{\varepsilon}(t)$ is absolutely continuous on $[A, B], \varepsilon k(t) / y_{\varepsilon}^{\prime}(t)$ can be regarded as an absolutely continuous function defined on $[A, B]$. Substituting $t=v_{\varepsilon}(s)$ into $(12)$ yields

$$
\begin{aligned}
w_{\varepsilon}\left(v_{\varepsilon}(s)\right) & =\varepsilon k\left(v_{\varepsilon}(s)\right) / y_{\varepsilon}^{\prime}\left(v_{\varepsilon}(s)\right) \\
& =\varepsilon k\left(v_{\varepsilon}(s)\right) v_{\varepsilon}^{\prime}(s) \quad \text { in }\left(s_{A}, s_{B}\right)
\end{aligned}
$$

and

$$
\lim _{s \rightarrow s_{A}, s_{B}} \varepsilon k\left(v_{\varepsilon}(s)\right) v_{\varepsilon}^{\prime}(s)=\lim _{s \rightarrow s_{A}, s_{B}} w_{\varepsilon}\left(v_{\varepsilon}(s)\right)=0 .
$$

Here we have used the fact that

$$
v_{\varepsilon}^{\prime}(s)=1 / y_{\varepsilon}^{\prime}\left(v_{\varepsilon}(s)\right)
$$

holds a.e. in $\left(s_{A}, s_{B}\right)$. Using the equalities $(9)_{\varepsilon}, s=y_{\varepsilon}\left(v_{\varepsilon}(s)\right)$, and the chain rule, we obtain

$$
\begin{aligned}
\varepsilon\left(k\left(v_{\varepsilon}(s)\right) v_{\varepsilon}^{\prime}(s)\right)^{\prime} & =w_{\varepsilon}^{\prime}\left(v_{\varepsilon}(s)\right) v_{\varepsilon}^{\prime}(s) \\
& =-\left(s g\left(v_{\varepsilon}(s)\right)-\varphi\left(v_{\varepsilon}(s)\right)\right) v_{\varepsilon}^{\prime}(s)-f\left(v_{\varepsilon}(s)\right)
\end{aligned}
$$

for almost all $s \in\left(s_{A}, s_{B}\right)$. When $s_{A}$ or $s_{B}$ is finite, the equalities (13) and (15) read $0=0$ on $\left(-\infty, s_{A}\right]$ or on $\left[s_{B},+\infty\right)$.

It follows from (13) and (15) that

$$
\begin{aligned}
\int_{a}^{b}\left(\varepsilon k\left(v_{\varepsilon}(s)\right) v_{\varepsilon}^{\prime}(s)\right)^{\prime} d s & =\int_{a}^{b} w_{\varepsilon}^{\prime}\left(v_{\varepsilon}(s)\right) v_{\varepsilon}^{\prime}(s) d s \\
& =w_{\varepsilon}\left(v_{\varepsilon}(b)\right)-w_{\varepsilon}\left(v_{\varepsilon}(a)\right) \\
& =\left.\left(\varepsilon k\left(v_{\varepsilon}(s)\right) v_{\varepsilon}^{\prime}(s)\right)^{\prime}\right|_{s=a} ^{s=b},
\end{aligned}
$$

where $[a, b]$ is any subinterval of $\left(s_{A}, s_{B}\right)$. Letting $a \rightarrow s_{A}$ and $b \rightarrow s_{B}$ in the above gives

$$
\int_{s_{A}}^{s_{B}}\left(\varepsilon k\left(v_{\varepsilon}(s)\right) v_{\varepsilon}^{\prime}(s)\right)^{\prime}=w_{\varepsilon}\left(v_{\varepsilon}\left(s_{B}\right)\right)-w_{\varepsilon}\left(v_{\varepsilon}\left(s_{A}\right)\right)=0 .
$$

Consequently, for all $s \in R$,

$$
\varepsilon k\left(v_{\varepsilon}(s)\right) v_{\varepsilon}^{\prime}(s)=\int_{-\infty}^{s}\left(\varepsilon k\left(v_{\varepsilon}(s)\right) v_{\varepsilon}^{\prime}(s)\right)^{\prime} d s .
$$

This shows that the left-hand side is absolutely continuous on $R$.

All the facts we have proved show that $v_{\varepsilon}(s)$ is a solution to the boundary value problem $(1)_{\varepsilon}-(2)$ and the proof is complete. 
Lemmas 7 and 8 imply that $v_{0}(s)$ must be a solution to the reduced boundary value problem $(1)_{0}-(2)$ and

$$
\begin{gathered}
y_{0}(t)=\Phi(B) / G(B), \quad \text { for all } t \in[A, B], \\
v_{0}(s)=A+(B-A) H\left(s-\frac{\Phi(B)}{G(B)}\right) \quad \text { for } s \in R .
\end{gathered}
$$

We can summarize the above results in the following

THEOREM 3. Under the hypotheses (I) and (II) or (I) and (II) ${ }^{\prime}$, the boundary value problem $(1)_{\varepsilon}-(2), \varepsilon \geq 0$, has a solution $v_{\varepsilon}(s)$. Moreover, as $\varepsilon$ tends to zero, the solution $v_{\varepsilon}(s)$ pointwise converges to the solution

$$
v_{0}(s)=A+(B-A) H\left(s-\frac{\Phi(B)}{G(B)}\right), \quad s \in R .
$$

5. The Riemann problem $(5)_{\varepsilon}-(6)$. In the last section we consider the Riemann problem $(5)_{\varepsilon}-(6)$. We shall call the function $u_{\varepsilon}(x, t)$ a solution to the Riemann problem $(5)_{\varepsilon}-(6)$ with $\varepsilon>0$, if it satisfies the following conditions:

(i) $u_{\varepsilon}(x, t)$ is a real-valued continuous function defined in the domain $R \times(0, \infty)$ with values in $[A, B]$,

(ii) $\lim _{t \rightarrow 0+} u_{\varepsilon}(x, t)=A+(B-A) H(x)$, for $x \in R$,

(iii) $\partial u_{\varepsilon}(x, t) / \partial x, \partial u_{\varepsilon}(x, t) / \partial t$, and $\partial\left(k\left(u_{\varepsilon}(x, t)\right) \partial u_{\varepsilon}(x, t) / \partial x\right) / \partial x$ exist a.e. in $R \times(0, \infty)$, and

(iv) the equality $(5)_{\varepsilon}$ hoids a.e. in $R \times(0, \infty)$.

If the solution $u_{\varepsilon}(x, t)$ converges to a limit, denoted by $u_{0}(x, t)$, pointwise on $R \times(0, \infty)$, as $\varepsilon$ tends to zero, then $u_{0}(x, t)$ is called a generalized solution to the Riemann problem $(5)_{0}-(6)$.

We are interested in similarity solutions

$$
u(x, t)=v(s), \quad s=\frac{x}{t} ;
$$

then we arrive at the boundary value problem $(1)_{\varepsilon}-(2)$.

THEOREM 4. Under the hypotheses (I) and (II) or (I) and (II) ${ }^{\prime}$, the Riemann problem $(5)_{\varepsilon}-(6)$ has a solution $u_{\varepsilon}(x, t)=v_{\varepsilon}(x / t)$. Moreover, as $\varepsilon$ tends to zero, the solution $u_{\varepsilon}(x, t)$ pointwise converges to the generalized solution

$$
u_{0}(x, t)=A+(B-A) H\left(\frac{x}{t}-\frac{\Phi(B)}{G(B)}\right)
$$

to the Riemann problem $(5)_{0}-(6)$.

Proof. The existence and convergence of the solution are an immediate consequence of Theorem 3.

Acknowledgment. The authors would like to thank Professor Wang Junyu for his helpful suggestions. 


\section{REFERENCES}

[1] C. M. Dafermos, Polygonal approximations of solutions of the initial value problem for a conservation law, J. Math. Anal. Appl. 38, 33-41 (1972)

[2] L. Holden and R. Høegh-Krohn, A class of $N$ nonlinear hyperbolic conservation laws, J. of Differential Equations 84, 73-99 (1990)

[3] O. A. Oleinik, Uniqueness and stability of the generalized solution of the Cauchy problem for a quasilinear equation, Uspekhi Mat. Nauk, Vol. 14, 165-170 (1959); Amer. Math. Soc. Transl. (2), 33, 285-290 (1963)

[4] J. Serrin and S. Varberg, A general charm rule for derivatives and change of variables formula for the Lebesgue integral, Amer. Math. Monthly 76, 514-520 (1969)

[5] J. Smoller, Shock Waves and Reaction-Diffusion Equation, Springer, New York, 1983

[6] Wang Junyu, The jump conditions for second order quasilinear degenerate parabolic equations, J. Partial Differential Equations 3, 39-48 (1990) 